\title{
“Pavlov's Buzz? Mobile Vibrations as Conditioned Rewards
}

\section{Contribution Statement}

Consumers spend much of their day engaged with their mobile devices. These devices emit hundreds of vibrations per day, triggered by anything from a new message or, more recently, adding an item to an Amazon shopping basket. Despite the prevalence of mobile vibrations, consumer research has not examined how and why consumers respond to mobile vibrotactile stimuli, nor their effect on decision-making. We contribute to the emerging literature on the technology-augmented consumer by providing a rigorous assessment of how different mobile vibrations are perceived, focusing on those evoking a reward response and which can alter mobile e-commerce consumer decision-making. We find evidence that mobile vibration is becoming an increasingly prevalent secondary reward that, due to a conditioning mechanism, can systematically influence consumers, particularly younger and more impulsive ones. These findings advance burgeoning psychology of technology research by bridging previously disparate consumer touch, haptic perception, and reward conditioning research streams. Our studies also make three main contributions for non-academic stakeholders.

First, we identify a mobile vibration that evokes a reward response in many consumers (400ms, Pilot study), providing actionable guidance for any firm interested in delivering a rewarding mobile experience. Second, we find that rewarding vibrations can boost online mobile shopping basket adds, offering an easy-to-implement way for firms to boost sales. The importance of these findings will likely continue to develop given that mobile spending is growing over three times faster than desktop spending (Comscore 2020a), and that online grocery shopping has become the top source of digital spending (Comscore 2020b). Together, our studies on vibrotactile feedback contribute to foundational work examining consumer decision-making in the context of haptic human-machine interactions. 


\begin{abstract}
People spend a large portion of their day interacting with vibrating mobile devices, yet how consumers respond to the vibrotactile sensations emitted by these devices, and their effect on consumer decision-making is largely unknown. Integrating recent work on haptic sensory processing and classical conditioning, the current research examines: (1) the relationship between vibration duration and reward response, (2) to what extent rewarding vibrations modify consumer decision-making, and (3) the underlying mechanism of this effect. We find that mobile vibrations of intermediate length (400ms) evoke a reward response, particularly among impulsive consumers, which in turn boosts purchasing in online shopping environments. We examine the impact of mobile vibrations on consumer decision-making in a variety of experimental settings, drawing on a diverse participant pool, leveraging both controlled experiments and a large, country-wide field experiment to assess theoretically and practically important boundary conditions. We further examine the mechanism of this effect, providing direct evidence that vibrations can influence consumers via classical conditioning, such that vibrations become rewarding due to their learned association with positively valenced mobile events. Our findings have important implications for the effective design of haptic interfaces in marketing and the role of mobile vibrations as a novel form of reward.
\end{abstract}

Keywords: vibration, haptics, mobile devices, consumer decision-making, reward 
Check the pocket or bag of nearly any person and you will find a vibrating mobile device. Recent estimates suggest that over $96 \%$ of US consumers now own a mobile phone (Silver and Cornibert 2019) and that mobile spending is growing over three times faster than desktop spending (16\% vs. 5\%; Comscore 2020a). The COVID-19 pandemic has accelerated this trend, boosting online shopping, particularly online grocery shopping, which in 2020 surpassed apparel as the top source of digital spending (Comscore 2020b). This fundamental shift in consumptive behavior demands that marketers adapt in order to manage the complex, interactive consumer experience mediated by a plethora of touchscreen devices (Shankar et al. 2010).

One increasingly prevalent, but understudied, stimulus in the realm of digital consumption is mobile vibrotactile feedback. Consumers now receive a nearly continuous stream of mobile notifications stemming from a diverse array of events such as messages from friends and family, calendar notifications, and operating system requests (Mehrotra et al. 2015). For many, this plethora of notifications is conveyed haptically via mobile vibration, leading to new phenomena such as "phantom vibrations" (i.e., largely benign vibratory hallucinations; Rosenberger 2015), which are experienced by nearly $90 \%$ of young adults (Drouin, Kaiser, and Miller 2012). In parallel, online retail and e-commerce firms have recently begun to deliver mobile vibrations to consumers in shopping contexts. For example, in 2019 Amazon updated their mobile application to make shopping cart adds trigger a vibration and large online fashion retailers such as ASOS and Zara Home also now leverage vibrational stimuli for shopping cart adds.

In addition to these online shopping companies, firms across industries are beginning to produce hardware that provides vibratory feedback in response to a variety of behaviors. For example, newer models of FitBit produce a vibration upon reaching a target number of steps (Vanhemert 2015), Lumo Lift promises to help you maintain good posture via vibrational 
reminders (Peppet 2014), and Pavlok users can set a bracelet to vibrate in sync with normative behaviors to encourage a healthy lifestyle (Constine 2014). Notably, these products and services use different kinds of vibrations (shorter or longer) for different purposes (goal completion, coaching, and training). Together, this renders a world in which we are experiencing an increasing number of vibrations in both personal and commercial contexts, yet it remains unclear how individuals subjectively respond to these different vibrations and what effect they might have on consumers.

The majority of prior work examining subjective responses to vibrational stimuli comes from the ergonomics and information systems literature (Cole, Mohammed, and Shanmugam 2015; Koskinen, Kaaresoja, and Laitinen 2008; Lee and Lim 2014; Saket et al. 2013). Only one academic study has directly examined the effects of mobile-generated vibration in the marketing domain, finding that a vibrational stimulus can boost the persuasiveness of social messages by increasing the perceived social presence of the communication (Hadi and Valenzuela 2019). Still unaddressed is how consumers perceive different kinds of mobile vibrations, whether they influence real consumptive choices, and how they might exert such influence.

In what follows, we first develop a novel theory building on upon prior work on touchmediated consumer decision-making in marketing (Brasel and Gips 2014, 2015; Hadi and Valenzuela 2019; Melumad and Pham 2020; Shen, Zhang, and Krishna 2016) and classical conditioning theory (Olson and Fazio 2001; Pavlov 1932). We then present the results of five studies, including a large-scale field experiment across an entire country designed to test our predictions. The results of these studies, conducted in a variety of experimental settings and across a range of participant pools, provide foundational evidence that a mobile vibration of intermediate duration (around 400ms) evokes a reward response (particularly for more impulsive 
consumers), which stimulates this specific vibration-paired consumer behavior, and that this effect can be explained by an underlying process of classical conditioning. We conclude by discussing how the current work expands research on the "psychology of touch" and the implications for the effective design of haptic interfaces in marketing.

Theoretical Background

Consumer touch and mobile technology. Touch is one of our five primary senses that facilitates our understanding of the world around us (Wolfe et al. 2006). Haptic information collected through touch profoundly influences consumer choice (Krishna and Morrin 2007; Peck and Terry L Childers 2003; Peck and Terry L. Childers 2003; Peck and Shu 2009; Peck and Wiggins 2006), including related psychological phenomena such as ownership and endowment (Peck and Shu 2009; Shu and Peck 2011). The influence of touch is in fact so powerful that merely touching a positively valenced object has been shown to affect persuasion, even if the touched feature provides no relevant information about the product (Peck and Wiggins 2006). Interestingly, consumers are often partially or completely unaware of the influence that touch sensations have on their evaluations and decisions (Crusco and Wetzel 1984; Krishna and Schwarz 2014).

With the rapid adoption and integration of mobile technology, much of our haptic stimulation now stems from interaction with mobile devices. Shopping on touchscreen devices, compared to shopping on a traditional computer, also increases psychological ownership of purchased products (Brasel and Gips 2014). Similarly, consumers looking for travel accommodation on a touchscreen interface (vs. traditional computer) search for more alternatives 
and have higher levels of anticipated satisfaction (Brasel and Gips 2015). Use of touchscreen devices also increases preference for affect-rich food items (Shen et al. 2016).

Subjective response to vibration. One critical, but underexplored, feature of touchsensitive devices is the mechanistic transfer of vibratory stimuli to users. Although we know from sensory perception research that the human hand has an extremely small sensory threshold for vibrotactile stimuli (as small as $0.2 \mu \mathrm{m}$ in displacement; Gescheider et al. 1999), much of prior research has examined vibration primarily in the context of ergonomics or how mechanically generated whole body vibration can cause injuries such as lower back problems (Bovenzi and Zadini 1992). Similarly, research examining mobile vibration has been largely limited to its use as an alerting stimuli (Saket et al. 2013) or the potential of vibration to convey multifaceted communication messages (Brown and Kaaresoja 2006; Sahami et al. 2008).

Among the distinguishing features of a vibrotactile stimulus, usability research suggests that the duration may be key to the response a vibration evokes. Early work found that very brief vibrational pulses are often not perceived (Kaaresoja and Linjama 2005), and that vibration duration is a key determinant of perceived intensity (Green 1976; Verrillo 1965). Preliminary evidence suggests that the duration of a mobile vibration may also affect perceptions of valence and arousal (Seifi and Maclean 2013; Yoo et al. 2015). However, this preliminary work does not address whether different mobile vibration durations evoke specific subjective responses, by what psychological mechanism these responses came about, nor how these differential responses affect downstream consumptive behavior. One recent study does suggest, however, that mobile vibration can affect perception of social messages. Hadi and Valenzuela (2019) found that pairing a mobile vibration with a health-promoting text message, resulted in higher self-reported 
levels of physical activity. The authors found evidence that adding a vibration to a mobile text message boosted the persuasiveness of the message by increasing perceived social presence.

While these initial findings indicate that mobile vibration can influence subjective response and behavior more broadly, they do not elucidate how consumers psychologically respond to mobile vibrations, to what extent this response varies as a function of the duration, nor the underlying mechanism that governs subjective response to mobile vibrations. Anecdotally, people tend to report that a vibration from their phone evokes an approach response, such as phone checking, but scholarly research examining the nuances of this response is lacking. Given that approach responses are very often triggered by reward-seeking (Simon et al. 2010), the question arises as to whether mobile vibration might be construed as a reward.

Mobile vibration reward response. A reward is typically conceived as being either primary (providing direct survival benefits such as food) or secondary (i.e., providing indirect benefits such as money; Sescousse et al. 2013). While the former tends to be intrinsically rewarding, the latter typically only becomes rewarding through learned association. We propose that mobile vibration has come to evoke a reward response due to classical conditioning (Figure 1), in which a behavior that is reinforced by a stimulus becomes more likely to occur in the presence of that stimulus (Gormezano and Moore 1966). As shown in Figure 1, positively valenced mobile events (such as a message from a friend) would initially be unconditioned stimuli that produced feelings of pleasure (i.e., the unconditioned reward response). This notion supported by studies showing that the majority of mobile event notifications are triggered by social messages (Mehrotra et al. 2015), which have been shown to activate reward areas of the brain (Sherman et al. 2016). Mobile vibration in this context functions effectively as a cue for the notification because it either co-occurs with the notification (during active mobile use) or 
precedes the notification (when the mobile is "locked"). Eventually this conditioned stimulus (mobile vibration) in insolation could theoretically evoke a reward response (conditioned response). Critically, this classical conditioning explanation requires that a person finds the initial unconditioned stimulus (i.e., the mobile event) to be positively valenced.

Figure 1. Conceptual Model of Mobile Vibrations as Conditioned Rewards

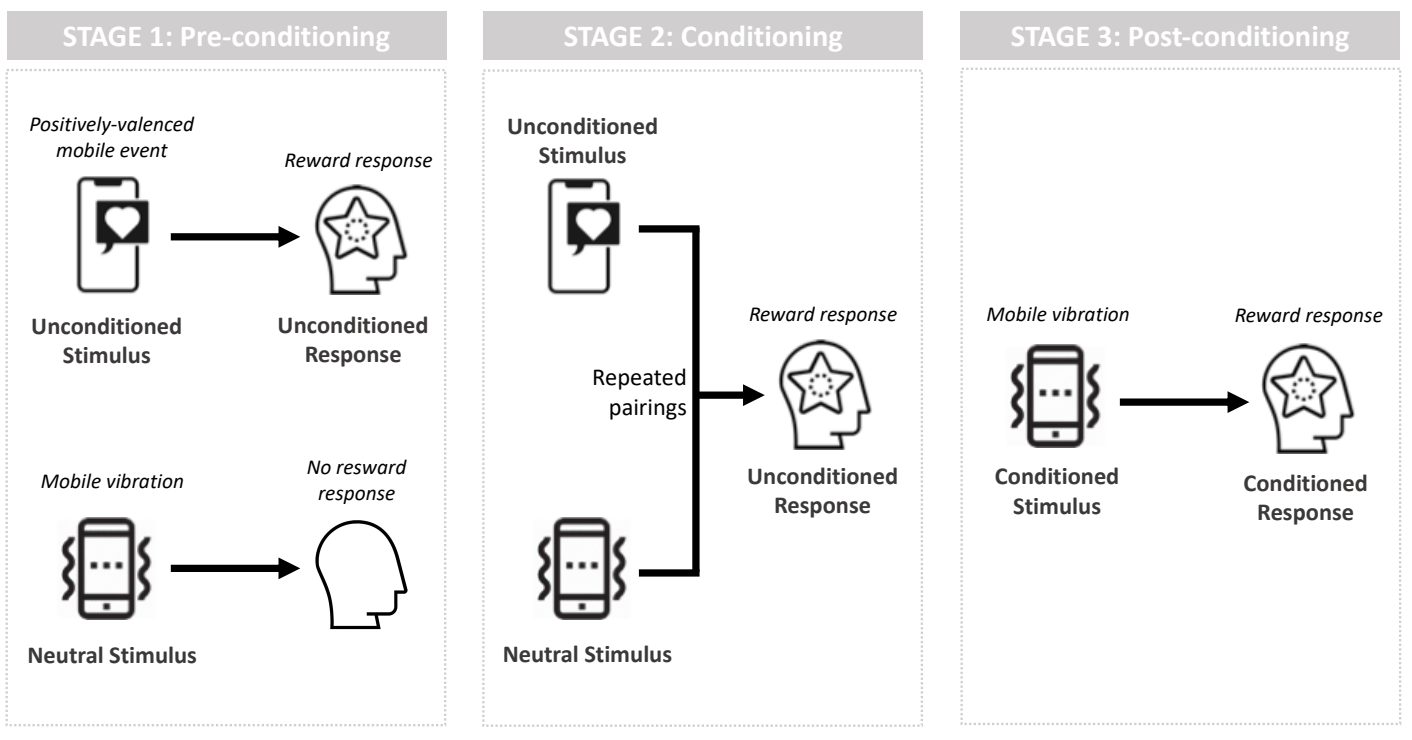

If certain mobile vibrations are rewarding, then following a large reward literature (Berridge 2000; Kelley 2004), such vibrations could in theory motivate behavior. Specifically, building on this prior work on classical conditioning and reward responsiveness, a key hypothesis of the current research is that pairing a rewarding vibration with a specific mobile behavior (such as adding an item to the shopping basket) will increase the frequency of that behavior.

Impulsivity, reward response, and consumption. The impact of mobile vibrations on reward perception is most likely not the same for all consumers. One key individual difference measure that has been shown to systematically affect reward conditioning is impulsivity (van den 
Akker et al. 2013). Impulsivity in a consumer context refers to the tendency to engage in unplanned purchases (Beatty and Ferrell 1998), which account for up to $62 \%$ of sales in certain contexts (Cheng et al. 2013). Individuals vary significantly in their trait level of impulsivity (Brown et al. 2006), which affects not only consumption (Davenport, Houston, and Griffiths 2012; Puri 1996), but also how people respond to a variety of rewards, such as money (Hampton et al. 2017; Wilmer et al. 2019) and food (Schag et al. 2013). Impulsivity has also been associated with variability in mobile technology interaction, with individuals who are more highly engaged tending to be more impulsive and more responsive to rewards (Hadar et al. 2015; Wilmer and Chein 2016a), possibly due to hyperconnectivity among reward areas of brain (Wilmer et al. 2019). Finally, impulsivity influences reward conditioning, with more impulsive individuals typically more easily influenced by conditioning paradigms (van den Akker et al. 2013; Gupta 1990). Building on this prior work on impulsivity and reward responsiveness, we further predict higher impulsivity will moderate the effect of vibrations on consumer purchasing outcomes such that consumers with higher levels of impulsivity will find mobile vibrations more rewarding than consumers with lower levels of impulsivity.

Together, this theoretical background led us to four overarching predictions: (1) vibration duration is a key determinant of vibration reward response (2) pairing a rewarding mobile vibration with a specific behavior increases the frequency of that behavior, (3) reward response to mobile vibrations is amplified for more impulsive consumers, and (4) that this effect is evoked through a classical conditioning process that can render a neutral vibrational stimulus rewarding if paired with a positively valenced mobile event. The exact nature of what constitutes a "rewarding" mobile vibration is operationalized and empirically assessed in the Study 1 and subsequently validated experimentally across all following studies. 


\section{OVERVIEW OF STUDIES}

Study 1 demonstrates that different vibration durations evoke differential subjective response, with an intermediate duration (400ms) maximizing reward response. We further show that reward response to intermediate vibration durations is stronger for more impulsive consumers and consumers with more positively valenced mobile events. Study 2 then shows that rewarding vibrations stimulate behavior, demonstrating that consumers press an add-to-cart touchscreen button more if it produces a rewarding vibration, and rules out novelty and arousal as alternative mechanisms. Study 3a leverages a highly controlled behavioral lab environment that extends the ecological validity of these findings to an online grocery shopping setting and demonstrates that vibrotactile feedback can boost purchasing, particularly for more impulsive consumers. Study $3 \mathrm{~b}$ replicates and extends these findings in a large-scale field experiment across an entire country, showing that mobile vibrations increase the number of purchased items and sales. Finally, in Study 4 we leverage a differential classical conditioning task to alter response to a neutral mobile vibration, providing direct evidence for our underlying theorizing and proposed classical conditioning mechanism. The findings of this study provide evidence that mobile vibrations evoke a reward response and affect consumer behavior due to prior repeated associations with positively valenced mobile events.

\section{STUDY 1}

The key objectives of the Study 1 were to examine how varying vibration duration influences reward response, and how this effect varies by consumer impulsivity and mobile event valence. 
Design and Procedure

We recruited 150 participants from Amazon Mechanical Turk (MTurk) to participate in a study on technology use. We excluded seven participants for failing a basic attention check, resulting in a final sample of $143\left(M_{\text {Age }}=31.2,60 \%\right.$ male $)$.

Hardware precheck. We advertised our study as only available to vibration-capable Android mobile device users and further included a hardware check to ensure that only eligible individuals participated. Specifically, we presented participants with an array of code-labeled buttons and instructed them to press each button, then enter the code from the button that produced a vibration in a field below (see Web Appendix A1.1 for details). We included this hardware check across all further online studies.

Vibration response questionnaire. We briefed participants on the general content of the study and instructed them to hold their mobile device in their hands for the duration of the study. In the first section, participants experienced a series of vibrotactile stimuli. These stimuli were created using customized jQuery code that allowed us to manipulate the vibration duration emitted by the mobile vibration motor, while holding intensity and all other features constant. Specifically, on each page, participants first experienced a vibration of a pre-defined duration. Vibration durations were presented in random order and were as follows (in milliseconds): 25, 50, 100, 200, 400, 800, 1600, and 3200; (Web Appendix A1.2; see https://virtulab.ch/durtest.html [Android operating system only]). Participants then self-reported their response to each vibrational stimulus on 7-point Likert scales (e.g., "This vibration is like a reward"). Specifically, based on prior research examining response to mobile vibrations (Yoo et al. 2015), participants indicated the extent to which they felt that a vibrational stimulus was: rewarding, arousing, unpleasant, punishing, stimulating, pleasant, tiring, and alerting. To counter 
order effects, both the presentation of the different vibration durations as well as all scale items within each vibration duration page were fully randomized.

Mobile device usage. We further collected an array of mobile usage measurements, each of which could conceivably relate to the previous set of vibration response measures. We measured the following features of participants' mobile usage: 24-hour overall usage, daily number of messages (any kind) sent and received, and daily phone checking frequency ("How often do you check your mobile device (any of them)?”; Wilmer and Chein 2016b). We included these particular measures of mobile usage and behavior because they have previously been associated with reward responsiveness and impulsivity (Wilmer et al. 2019; Wilmer and Chein 2016b) and therefore might explain variance in mobile vibration response. Second, as our theoretical model (Figure 1) posits that mobile vibration conditioning entails an association with a positively valenced mobile event, we also asked participants to report the valence of their daily notifications ("What percentage of the notifications that you receive are welcome?"; see Web Appendix A1.4 for the full set of measures).

Trait measures. We included a set of individual difference trait measurements to test our theorizing. First, we captured impulsivity using the Barratt Impulsiveness Scale (e.g., "I do things without thinking”, $\alpha=.82$; Patton, Stanford, and Barratt 1995). Following previous research (Steinberg et al. 2008; Wilmer et al. 2019), we selected the 18 items from the full 30item questionnaire with greatest specificity with respect to impulsive behavior. As previously described, we anticipated that impulsivity would enhance consumers' perception of mobile vibration response. Second, we assessed reward sensitivity, following Wilmer and colleagues 2019 (e.g., "When good things happen to me, it affects me strongly", $\alpha=.73$ ). Next, given that individual differences in touch proclivity might influence response to mobile vibration, we also 
assessed participants' Need for Touch (e.g., "I feel more confident making a purchase after touching a product", $\alpha=.93$; Elhai et al. 2016; Peck and Childers 2003a). Finally, we collected participants' demographic information (age, gender, level of education, and annual income).

Results

Vibration response and vibration duration. Figure 2 provides a summary of the main results (full set of measures in Web Appendix D1.1), illustrating that participants responded to vibrational stimuli significantly differently depending upon the duration and the response measure. A series of linear mixed-effects models predicting each response confirmed this pattern. Following Gelman and Hill (2007), we first log-transformed vibration duration (initially ranging from 25 to 3200 ) to rescale the variable to be comparable with response measures.

First, we modeled the extent to which reward response varied as a function of vibration duration, accounting for repeated observations by including participant as a random effect. We included the square of duration as an additional fixed effect to test for potential quadratic effects (see Figure 2). We find both a significant positive effect for vibration duration on reward response $\left(\beta_{\text {VibrationDuration }}=3.05 ; t(1,1141)=7.47, p<.001\right)$ and, most importantly, a significantly negative squared effect of vibration duration on reward response $\left(\beta_{\text {VibrationDuration }}{ }^{2}=-.63\right.$; $t(1,1141)=-7.57, p<.001)$, confirming the quadratic relationship apparent in Figure 2. In contrast, there was an approximately positive linear effect of vibration duration on both arousal $\left(\beta_{\text {VibrationDuration }}=.64 ; t(1,1141)=8.04, p<.001\right)$ and punishment $\left(\beta_{\text {VibrationDuration }}=.68\right.$;

$t(1,1141)=7.73, p<.001)$. 
Figure 2. Intermediate Vibration Duration Maximizes Reward Response (Study 1)
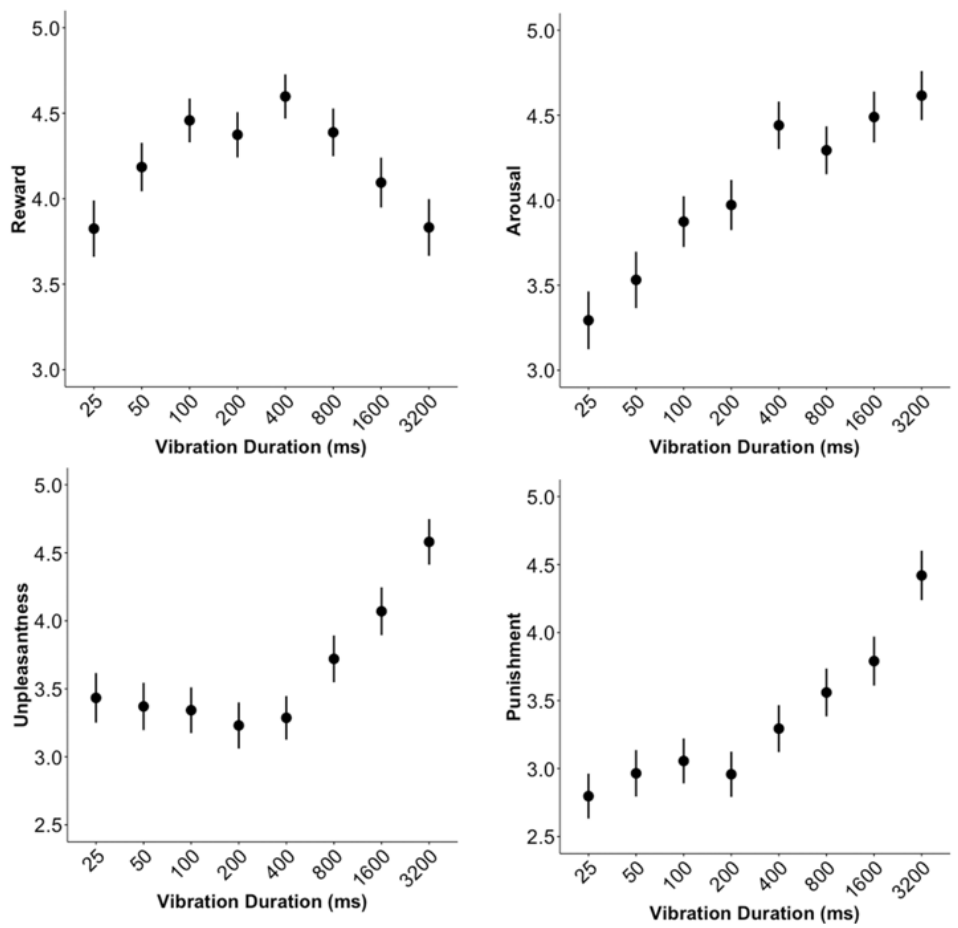

Next, we examined which vibration duration evoked the strongest reward response.

Planned contrasts demonstrated that the reward response to the $400 \mathrm{~ms}$ vibration $\left(M_{\text {Reward }}=4.36\right)$ was significantly stronger than all other durations (all $p<.05)$, except for $800 \mathrm{~ms}\left(M_{\text {Reward }}=4.24\right.$, $p=.80$ ). To determine whether $400 \mathrm{~ms}$ was indeed more rewarding than other stimulus durations, we compared reward response to $400 \mathrm{~ms}$ to that of both longer durations ( $800 \mathrm{~ms}$ or longer) and shorter durations (200ms or shorter). Reward response to the intermediate duration of $400 \mathrm{~ms}$ was significantly stronger relative to both longer durations $(t(1,1141)=-2.38, p<.05)$ and to shorter durations $(t(1,1141)=-2.01, p<.05)$. Consistent with the findings on reward response, we find that the $400 \mathrm{~ms}$ vibration was perceived as significantly less unpleasant than the $800 \mathrm{~ms}$ vibration $\left(M_{400 \mathrm{msUnpleasantness}}=3.29\right.$ vs. $\left.M_{800 \mathrm{msUnpleasantness}}=3.72, t(1,1141)=-1.83, p<.05\right)$. Finally, response to $400 \mathrm{~ms}$ vibration did not differ significantly from the $800 \mathrm{~ms}$ vibration in terms of either 
arousal or punishment (both $p>.40$ ). Together these analyses indicate that $400 \mathrm{~ms}$ evoked the most positive and rewarding response.

Impulsivity and valence as drivers of reward response. First, we examined to what extent impulsivity and notification valence moderated the functional relationship between vibration duration and vibration reward response. Consistent with our theorizing, we find that both impulsivity and notification valence were significant moderators such that people who were higher in impulsivity $\left(\beta_{\text {Imp } \times \text { Duration }}=.02 ; t(1,1141)=2.05 p<.05\right)$ or reported more positive mobile notification valence $(\beta$ Valence $\times$ Duration $=.63 ; t(1,1141)=2.68, p<.01)$ also exhibited a stronger reward response to vibrations across the durations. These findings provide evidence that vibration reward response peaks at an intermediate duration of $400 \mathrm{~ms}$ and, consistent with our theorizing, that impulsivity and valence of mobile notifications moderate the strength of mobile vibration reward response.

Finally, nonparametric bivariate Spearman's rank correlation coefficients further revealed that both general and peak vibration reward response were most strongly correlated with impulsivity $\left(\mathrm{r}_{\text {MeanReward }}=.57,95 \%-\mathrm{CI}=[.44, .67], p<.001 ; \mathrm{r}_{400 \mathrm{msReward}}=.43,95 \%-\mathrm{CI}=[.41\right.$, .54], $p<.001)$. Stronger vibration reward response also positively correlated with the positive valence of mobile notifications $\left(\mathrm{r}_{\text {MeanReward }}=.29,95 \%-\mathrm{CI}=[.11, .41], p<.01 ; \mathrm{r}_{400 \mathrm{msReward}}=.22\right.$, $95 \%-\mathrm{CI}=[.09, .37], p<.01)$, as well as overall mobile usage $\left(\mathrm{r}_{\text {MeanReward }}=.24,95 \%-\mathrm{CI}=[.03\right.$, $\left..36], p<.01 ; \mathrm{r}_{400 \mathrm{msReward}}=.30,95 \%-\mathrm{CI}=[.08, .40], p<.01\right)$. A full table of correlations including other factors (need for touch, reward sensitivity, phone checking, notification count, education, and income) can be found in Web Appendix D1.2. 
Table 1. Bivariate Spearman's correlations between vibration response, impulsivity, and notification valence (Study 1)

\begin{tabular}{|c|c|c|c|c|c|c|c|c|}
\hline & $\begin{array}{c}\text { Vibration } \\
\text { Mean } \\
\text { Reward }\end{array}$ & $\begin{array}{c}\text { Vibration } \\
400 \mathrm{~ms} \\
\text { Reward } \\
\end{array}$ & Impulsivity & $\begin{array}{l}\text { Mobile } \\
\text { Usage }\end{array}$ & $\begin{array}{c}\text { Notification } \\
\text { Valence }\end{array}$ & Age & Mean & SD \\
\hline Vibration Mean Reward & - & & & & & & 3.99 & 1.55 \\
\hline Vibration $400 \mathrm{~ms}$ Reward & $.78^{* * *}$ & - & & & & & 4.36 & 1.89 \\
\hline Impulsivity & $.57^{* * *}$ & $.43^{* * *}$ & - & & & & 12.80 & 6.01 \\
\hline Notification Valence & $.29^{* * *}$ & $.22^{*}$ & $.29^{* * *}$ & .12 & - & & 43.00 & 26.39 \\
\hline Mobile Usage & $.24^{* *}$ & $.30^{*+*}$ & $.29^{* * *}$ & - & & & 3.77 & 2.86 \\
\hline
\end{tabular}

Discussion

The findings of the Study 1 provide evidence for our key hypothesis that vibration duration affects reward response. Critically, we found that vibrations of intermediate duration (400ms) are perceived as maximally rewarding. Importantly, because we randomized the presentation order of the vibrations, this finding cannot be explained by simple order or demand effects. We provide further experimental evidence contrary to such alternative explanations in Studies 2 and 3a. We also demonstrated that that vibration reward response is magnified by higher impulsivity and more positive notification valence. Finally, we also find initial, correlational support for the idea that classical conditioning explains the relationship between mobile vibration exposure and reward response (formally tested in Study 4) by demonstrating a link between vibration reward response and mobile notification valence.

\section{STUDY 2}

The objectives of Study 2 were twofold. First, building on the findings of the Study 1 (i.e., that a $400 \mathrm{~ms}$ vibration maximizes reward response), Study 2 provides a direct test of our theorizing by examining whether pairing a rewarding vibrational stimulus with a simple action 
(pressing a button on a touchscreen) promotes that action. Second, the current study further examines whether the predicted effect is driven by vibration reward response (per our theorizing) or a simple arousal or novelty.

\section{Design and Procedure}

We recruited 400 participants from an online panel (Mturk) and randomly assigned them to a between-subjects design with three conditions (see next paragraph for details). Eleven participants failed the attention check, leaving $389\left(M_{\mathrm{Age}}=31.99,59 \%\right.$ male $)$ as the final sample. After receiving device handling instructions mirroring those of the Study 1, participants completed a "Box Task".

Box task. This task was designed to mimic the action of adding an item to a shopping cart in a tightly controlled context. Specifically, we presented participants with a button below a picture of a box, and the following task instructions: "In this task, there is a box to which you can add items by clicking on the button below. Add as many items to the box as you like. After 1.5 minutes of doing this task, you will be able to click below to continue to the next part of the study." Participants were free to add as many times as they liked during this fixed period, with no other incentive or rationale given. In all conditions, when a participant added an item to the box, they received an identical text pop-up confirmation, and an updating item counter displayed how many items were currently in the box (see Web Appendix A2.1 screenshot). Participants were randomly assigned to one of three experimental conditions that determined the kind of vibrational feedback item adds triggered: no vibration $(n=130), 400$ ms vibration $(n=129)$, or $3200 \mathrm{~ms}$ vibration $(\mathrm{n}=130)$. We selected durations of $400 \mathrm{~ms}$ and $3200 \mathrm{~ms}$ because Study 1 showed that the $400 \mathrm{~ms}$ mobile vibration evoked a stronger reward response but less arousal than 
3200ms (Figure 1). Aside from conditional variation in vibration feedback, visual feedback was identical. The main dependent variable in this study was the number of items that participants added to the box.

Post-task measurements. After the Box Task, participants completed an abbreviated version of the vibration response questionnaire (see Study 1). Specifically, participants reported their response to the two mobile vibrations used in the vibration conditions $(400 \mathrm{~ms}$, and $3200 \mathrm{~ms}$ ). Participants then reported their subjective reward and arousal responses to the vibrations as in the Study 1. To assess to what extent the current findings are driven by mere differences in novelty perception, we included a composite measure of how familiar each vibration was to participants (e.g., "This mobile vibration is familiar to me", $\alpha=.73$; adapted from Park and Lessig 1981; Web Appendix A1.3). Lastly, participants completed a demographic questionnaire.

Results

Item Count. A Kruskal-Wallis one-way ANOVA by rank revealed a significant effect of vibration duration on item count $\left(\chi^{2}(2, \mathrm{n}=389)=10.74, p<.01, \eta^{2}=.03\right)^{1}$. A Dunn Test with Benjamini-Hochberg correction (Thissen, Steinberg, and Kuang 2002) for multiple comparisons revealed that the $400 \mathrm{~ms}$ condition led to a significantly greater number of items added compared to both the $3200 \mathrm{~ms}$ condition $\left(M_{400 \mathrm{~ms}}=33.06\right.$ vs. $\left.M_{3200 \mathrm{~ms}}=19.85, p<.001\right)$, and the control condition $\left(M_{\text {Control }}=24.93, p<.05\right)$. These comparisons are depicted in Figure 3. The item count in the $3200 \mathrm{~ms}$ condition and control was not statistically different after correcting for multiple

\footnotetext{
${ }^{1}$ We conducted a nonparametric test (Gastwirth, Gel, and Miao 2009) because there was significantly different homogeneity of variance between conditions $(F(2,386)=11.53, p<.001)$. For comparison, we also conducted a traditional one-way ANOVA with post-hoc comparisons, which produced analogous results (Web Appendix B2).
} 
comparisons $(p=.66)$. That is, the highest arousal condition ( $3200 \mathrm{~ms})$ did not even outperform the control no vibration condition, despite being highly arousing.

Figure 3. Number of items added to box by condition (Study 2)

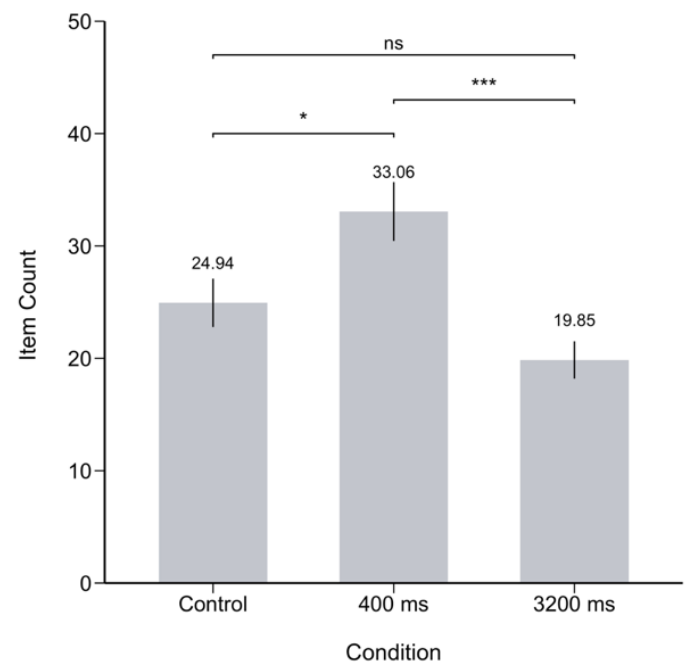

Notes: ${ }^{* * *}=p<.001,{ }^{*}=p<.05, n s=$ not significant. Error bars $=+/-1$ SEs.

Vibration reward response. Across all conditions, the $400 \mathrm{~ms}$ vibration induced a stronger reward response than either longer or shorter vibrations (all $p \mathrm{~s}<.001)$ replicating our Study 1 finding. We also find that mean reward response did not differ between conditions $(F(2,386)=.89, p=.41)$, indicating that differences in item count was not driven by variable exposure to mobile vibrations in the Box Task.

Alternative Mechanisms. First, we address the possible role of novelty effects. The $400 \mathrm{~ms}$ vibration was rated as significantly less novel $\left(M_{400 \mathrm{msFamiliarity}}=15.00\right)$ than the $3200 \mathrm{~ms}$ vibration $\left(M_{3200 m s F a m i l a r i t y}=11.53\right)$, as revealed by a planned contrast $(t(1,387)=10.45, p<.001)$. Given that participants added more items in the $400 \mathrm{~ms}$ (vs. $3200 \mathrm{~ms}$ ) condition, this finding runs directly contrary to a novelty explanation and suggests instead that greater familiarity explains the current set of findings. Furthermore, our results reveal that familiarity (i.e., lower novelty) 
positively correlated with mobile vibration reward response $(\mathrm{r}=.27,95 \%$ - $\mathrm{CI}=[.17, .36], p<.001)$. In other words, the more familiar the mobile vibration, and the closer it matched the vibration duration on a participant's personal mobile, the more rewarding they found that vibration. Associations of mobile vibrations with positively valenced mobile events was also related to stronger mobile vibration reward response $(\mathrm{r}=.44,95 \%-\mathrm{CI}=[.36, .52], p<.001)$. Together, these findings suggest that prior exposure to mobile vibrations linked to positive events drives reward response. We formally test the role of conditioning involving positively valenced mobile events as a driver of vibration reward response in Study 4.

\section{Discussion}

Study 2 demonstrates that pairing a rewarding mobile vibration with a specific behavior increases the propensity for that behavior. Specifically, we show that a $400 \mathrm{~ms}$ mobile vibration boosts item count in a tightly controlled item adding task. These findings also provide evidence that arousal and novelty cannot explain the current set of findings. On the contrary, we find that participants added significantly fewer items in the longer vibration condition (higher in both arousal and novelty) than in the intermediate-length vibration condition (lower arousal and novelty, but higher reward), consistent with our theorizing. A follow-up study also shows that this effect persists even within participant, such that when given a choice between two add-tobox buttons participants add more items using the button that produces a rewarding intermediate vibration relative to one that produces no vibration (full write-up in Web Appendix C1). 


\section{STUDY 3A}

The previous studies demonstrated that a $400 \mathrm{~ms}$ vibration evokes a reward response (Study 1 and Study 2), which increases consumer propensity to add items to a box (Study 2). Study 3a was designed to test three further propositions. First, we test if exposure to vibrotactile stimuli can influence item adds and shopping basket totals in a setting of high ecological validity (i.e., in a typical online shopping environment). Second, based on the preceding findings linking impulsivity and reward response, we test whether a rewarding vibrotactile stimulus (400ms) more strongly affects consumers with higher (vs. lower) levels of impulsivity. Third, we test the possibility that the observed effects are influenced by artefacts of online panel recruitment, including selection bias and device heterogeneity, by conducting the study in a behavioral lab environment and holding the type of device constant across participants and experimental conditions.

\section{Design and Procedure}

We recruited 138 participants $\left(56 \%\right.$ men; $\left.M_{\mathrm{Age}}=23.1\right)$ for a lab study. Unlike the previous studies that required pre-informing participants of the use of vibration in the study, we advertised Study 3 a more vaguely as a "Shopping Study". Upon entering the behavioral lab, we welcomed participants and retained their personal mobile devices for the duration of the study. Participants sat at partitioned cubicles, each with a Samsung Galaxy Tab 10 that was identical in terms of hardware and software, with all settings held constant such that there were no differences in display features such as screen luminance and brightness. Each study session was pseudo-randomly assigned to be either a control or vibration session. For vibration sessions, the 
vibration capability of the devices was activated, while for the control sessions, the vibration capability of the devices was deactivated. The setup between conditions was otherwise identical.

Online grocery shopping task. We built a grocery shopping web portal using customized PHP code within a Laravel v5.5 platform (www.laravel.com). We based the design of our experimental shopping portal on that of a large online grocery shopping website: all product images were borrowed from this website and we categorized our products into separate pages in a similar fashion. The task contained 6 pages, grouped by category (fruits and vegetables, dairy, meat and fish, sweet and salty snacks, breads and grains, and household items). Each page contained 30 items (180 items in total). Items were displayed in rows, each with a picture, and price in Swiss Francs $\left(\mathrm{CHF}^{2}\right)$, with an add-to-cart button below each item. We also included three discount items on each page both because this is common in real online markets and because discounts can evoke greater impulse purchasing (Zhou and Wong 2004). Across all items and pages, discount items were always $20 \%$ less than the original price, rounded to the nearest five cents. By design, all participants were constrained to move through the shopping task sequentially with "next" and "previous" buttons, but were free to iterate between pages as they wished. When a participant added an item to their cart, a shopping cart navigation bar at the top of the screen updated to indicate their current cart total and the number of items in their cart.

In the control condition, adding an item updated the cart count and basket total, but did not produce any other feedback. In the vibration condition, clicking the add-to-cart button had the same visual feedback as in the control, but also triggered a rewarding vibration of intermediate duration $(400 \mathrm{~ms})$, mirroring the setup of preceding studies. As previously noted, aside from this difference in vibrotactile feedback, all other elements of the two conditions were

\footnotetext{
21 CHF is approximately 1 USD.
} 
held constant. After the shopping task, participants were taken to a checkout page in which they could modify items in their cart, as well as return to previous pages, before finally submitting their order (a screenshot of this shopping task can be found in the Web Appendix A2.2). The key dependent measures in Study 3a were the total number of items added, the number of discounted items added, and shopping basket totals.

Post-task measurements. After completing the grocery shopping task, participants responded to an array of self-report measures, drawn partly from those used in Studies 1 and 2. For trait measures, we assessed impulsivity and demographics. Finally, to directly assess consumers' reward response to vibrotactile stimuli in the vibration condition ${ }^{3}$, participants were exposed to a battery of vibration durations. These measures included items from the Study 1 as well as an additional item of reward response ("This vibration makes me feel good"), which we combined with the reward and pleasant items to create a composite mobile vibration reward response measure (see Web Appendix A1.6 for further details), inspired by related measures of trait-level reward responsiveness (Van den Berg, Franken, and Muris 2010; Carver and White 1994). We refer to this composite measure of reward as "vibration reward response" in this and all subsequent studies and analyses. We also continued to examine differences in mobile reward response both at the most rewarding duration (400ms) as well as more generally, by averaging reward response across durations. Finally, we measured individual differences in grocery shopping behavior to ensure any such between-group differences did not confound our findings (see Web Appendix A1.7-1.8 for details).

\footnotetext{
${ }^{3}$ As the control group completed the study using devices with vibration disabled for the entire study, they did not complete the post-task vibration response battery.
} 
Results

Extending the findings of Study 2 to a more ecologically valid setting, consumers in the vibration condition added a significantly more items to their shopping cart $\left(M_{\text {VibrationTotalItemCount }}=18.64\right.$ vs. $\left.M_{\text {ControlTotalItemCount }}=15.75 ; F(1,138)=5.95, p<.05, \eta^{2}=.04\right)$. As expected, item count was significantly correlated with shopping basket totals $(\mathrm{r}=.91,95 \%$ $\mathrm{CI}=[.87, .93], p<.001)$, which were also significantly higher in the vibration group $\left(M_{\text {VibrationBasketTotal }}=\mathrm{CHF} 72.78\right.$ vs. $M_{\text {ControlBasketTotal }}=\mathrm{CHF} 57.84 ; F(1,138)=10.01, p<.001, \eta^{2}=$ .07 ; see Figure 4).

Figure 4: Total item count (Study 3a)

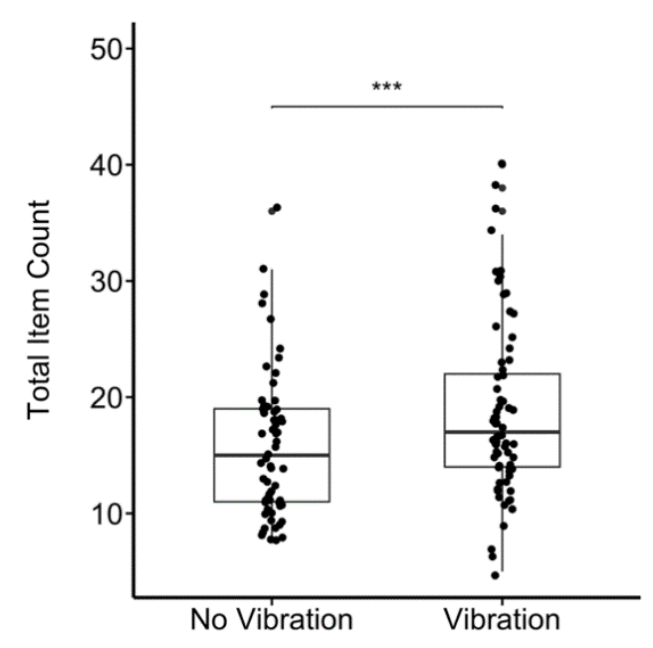

Notes: The effect of mobile vibration on number of purchased items. ${ }^{* * *}=p<.001$.

Looking at the number of discounted items purchased, a robust regression model ${ }^{4}$ further revealed that vibrotactile feedback also produced a consistent effect in the same (positive) direction with marginally more discount items added relative to the control group

\footnotetext{
${ }^{4}$ Here we use robust regression due to the presence of several outliers (1.5 times the interquartile range) in the count of discount items (Huber 1973).
} 
$\left(M_{\text {VibrationDiscountItems }}=4.30\right.$ vs. $\left.M_{\text {ControlDiscountItems }}=3.54 ; t(1,138)=1.71, p=.09\right)$. As expected, the number of discount items positively correlated with total number of items $(r=.36,95 \%-C I=[.20$, $.51], p<.001)$, as well as overall basket totals $(\mathrm{r}=.46,95 \%-\mathrm{CI}=[.30, .59], p<.001)$. Finally, the vibration and control groups did not differ on any of the general shopping, mobile, demographic, or trait variables measured (all $p \mathrm{~s}>.20$; Web Appendix A3.1), highlighting that the above effects cannot be attributed to ex-ante group differences.

Reward response, impulsivity, and notification valence. Next, we tested whether the current effects vary as a function of individual differences in impulsivity and to what extent consumers exhibit a reward response to mobile vibrations. In line with our prediction, we indeed find that consumers who were more impulsive and had a stronger vibration reward response also purchased more items in the shopping task $\left(\beta_{\text {Imp } \times \text { Reward }}=.29, t=1.95, p=.05\right)$. The main effect of impulsivity was marginally significant $\left(\beta_{\mathrm{Imp}}=.40, t=1.80, p=.08\right)$, while the simple main effect of reward response was non-significant $\left(\beta_{\text {Reward }}=.75, t=1.20, p=.23\right)$. The magnitude of these effects increased $\left(\beta_{\mathrm{Imp} \times \text { Reward }}=.33, t=2.21, p=.03 ; \beta_{\mathrm{Imp}}=.51, t=2.17, p=.03\right)$ after controlling for differences in general mobile phone usage (phone checking, notification count), general shopping frequency, and age (all $p s>.19$ ). Similarly, we find that consumers reporting higher positive valence for their mobile notifications had a stronger vibration reward response also tended to purchase more items $\left(\beta_{\text {Valence }}=.19, t=2.33, p<.05 ; \beta_{\text {Valence } \times \text { Reward }}=.08, t=2.45\right.$, $p<.01)$. Controlling for the previously mentioned factors did not affect the direction or magnitude of this effect (all $p s>.63$ ). Figure 5 further illustrates these results (based on a median split for illustrative purposes), showing that greater reward response to vibrations was associated with a significant increase in the number of items added during the shopping task for more impulsive consumers and those with higher notification valence, while reward response had no 
effect on the number of items added for less impulsive consumers and those with lower notification valence.

Figure 5: Moderating role of impulsivity and notification valence (Study 3a)
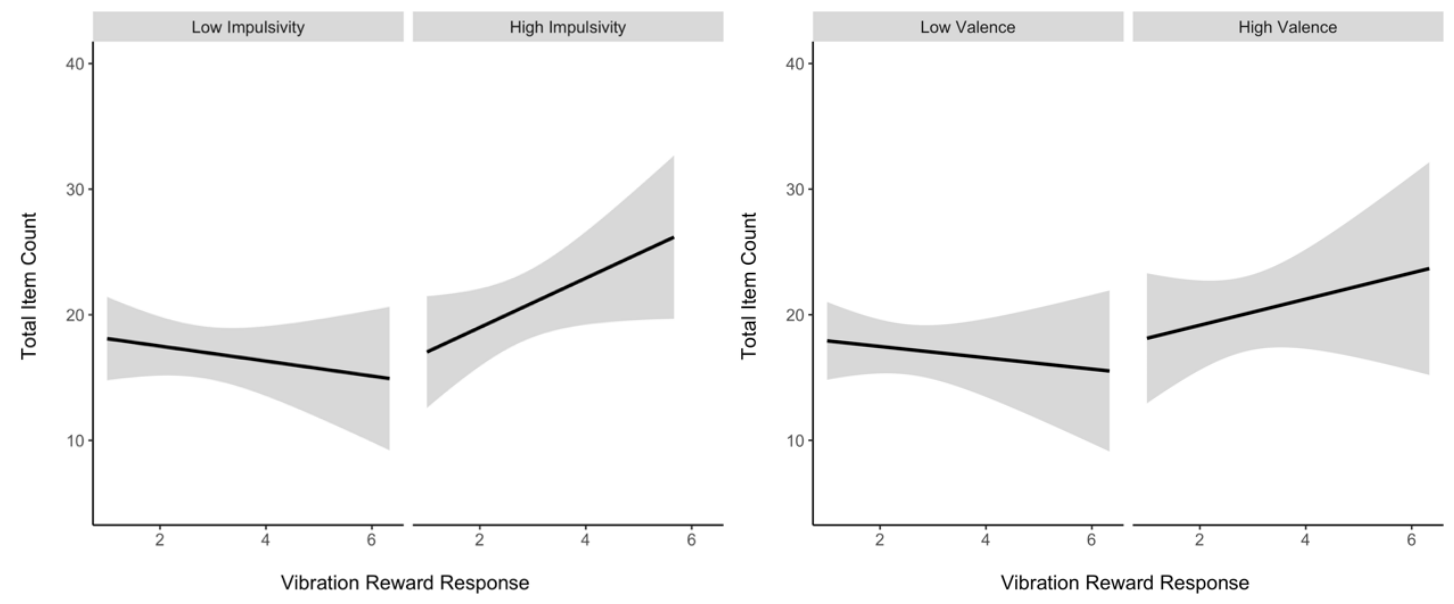

Robustness tests. Although we provided evidence that vibrotactile feedback alters behavior because it evokes a reward response, we wanted to rule out that this effect could have been driven by a simple novelty account. Conceptually replicating our previous finding (Study 2), we find that higher familiarity, not novelty, relates to vibration reward response. Specifically, we found that vibration reward response positively correlated with vibration familiarity both for $400 \mathrm{~ms}$ vibrations $\left(\mathrm{r}_{400 \mathrm{msReward}}=.59,95 \%-\mathrm{CI}=[.44, .70], p<.001\right)$ and on average across vibration duration $\left(\mathrm{r}_{\text {MeanReward }}=.54,95 \%-\mathrm{CI}=[.36, .68], p<.001\right)$.

\section{Discussion}

Study 3 a provided evidence that vibrotactile stimuli can alter consumer decision-making in an ecological mobile shopping environment. We found that delivering a rewarding vibration to participants for adding an item to their cart increased the quantity of both overall and discount 
items added, as well as total sales. We provided further evidence that the effect of vibrational stimuli increases in magnitude for consumers who exhibit higher levels of impulsivity (or mobile notification valence) and stronger responses to vibrational rewards. Finally, this study provided further evidence counter to the explanation that participants purchased more in the vibration condition because they found the vibration novel, showing that the prior exposure to mobile vibration is in fact associated with a stronger vibration reward response.

\section{STUDY 3B}

In Study 3b, we conducted a large-scale field experiment $(N=6,866)$ in cooperation with a major grocery store across an entire country to test the real-world validity of the base effect observed Study2a (i.e., that mobile vibrations can lead to more item adds and higher sales). Specifically, we facilitated the modification of the firm's mobile-based grocery shopping app to include rewarding mobile feedback (i.e., $400 \mathrm{~ms}$ ) for shopping cart adds for a subset of their customers. We anticipated that, consistent with Study 3a, participants in the vibration condition (vs. control group) will add more items and spend more per order. We expect a smaller effect in this field study (vs. Study 3a) given uncontrollable hardware and software factors (e.g., participants who have vibration turned off entirely) as well as our inability to control for important individual differences (e.g., impulsivity).

Design and Procedure

Working in cooperation with a major European grocery store, we conducted an anonymous, randomized field experiment drawing on regular online grocery shoppers of the company. We worked with this firm to develop an updated version of their mobile grocery 
shopping app for their customer base that included custom-made code that triggered vibration upon clicking the add-to-cart button (as in Study 3a). Customers were randomly assigned to either receive rewarding (400ms) vibrotactile feedback for shopping cart adds in the vibration condition or no vibrotactile feedback in the control condition. Participants were assigned to a condition according to whether their unique customer ID number was odd or even. In other words, consumers in both conditions used an app that was identical except for vibrotactile feedback for shopping cart adds. The initial timeseries dataset included all purchases (using any Android or iOS mobile device) made during the data collection period of one week. The key dependent measures provided by our industry partner were number of items purchased and net sales (gross sales minus sales returns, sales allowances, and sales discounts). We were then able to analyze individual-level basket sales, but for privacy and customer protection reasons, we were not able to analyze the item structure per basket.

We conducted three main data preprocessing steps (full preprocessing flow is depicted in Web Appendix A3.2). First, we removed 206 entries that were missing data (including age, gender, or device OS). Second, we selected only consumers who made fewer than three orders in the one-week period. This second step was per the advice of our industry partner who informed us that many online grocery orders come from business representatives making frequent and predetermined orders, which would be very resistant to our subtle vibration intervention and could therefore skew our results. We then calculated our two key dependent variables: number of items per order, and net sales per order, which were analogous to our measures from previous studies. We then examined these key variables, excluding 805 customers who made orders that were extreme outliers (greater than 3 standard deviations above the mean) in terms of their item count or net sales. Most importantly, we found no differences between experimental conditions 
in either item count or net sales in the excluded participants (details in Web Appendix A3.2). The final sample was $6,866,\left(M_{\mathrm{Age}}=43.90\right)$ which did not significantly differ between condition on any demographic trait or based on our exclusion criteria (all $p \mathrm{~s}>.85$ ). We statistically describe this final sample by condition in the Web Appendix A3.3. In what follows, we first examine the main effect of vibration condition on item count and net sales, and then conduct subsequent robustness tests that account for age, gender, and device operating system.

Results

Replicating our lab-controlled Study 3 a findings in a setting with real economic consequences, participants in the vibration condition purchased significantly more items per $\operatorname{order}\left(M_{\text {ControlItems }}=53.15\right.$ vs. $\left.M_{\text {VibrationItems }}=54.60, F(1,6864)=5.58, p<.05\right)$, which resulted in higher net sales $\left(M_{\text {VibrationSales }}=210.15\right)$ relative to the control condition $\left(M_{\text {ControlSales }}=206.88\right.$; $F(1,6864)=4.00, p<.01)$. In other words, consumers in the vibration condition added on average 1.5 more items per order to their final basket. As detailed further in the discussion, we attribute this smaller effect in this field study to uncontrollable factors. These results are illustrated in Figure 6. Item count positively correlated with net sales $(\mathrm{r}=.56,95 \%-\mathrm{CI}=[.54$, .57], $p<.001)$. This correlation is likely weaker than the correlation in our lab study between item count and sales $(r=.91)$ because net sales is less directly related to item count than is gross sales (not available in this study). 
Figure 6. Effect of mobile vibration on item count (Study 3b)

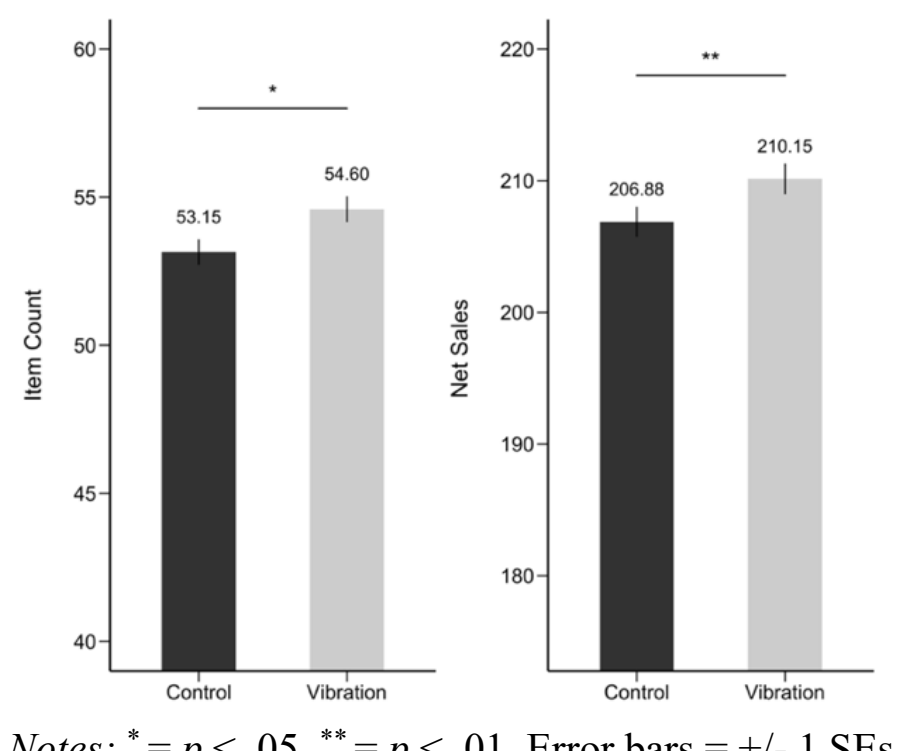

Next, we examined the robustness of these findings by including age, gender, and device operating system in multiple regression analyses. For item count, the overall regression model was significant $(F(4,6861)=41.95, p<.001)$, such that vibration condition $(\beta=1.56, t(6861)=2.52, p<.01)$, age $(\beta=-.23, t(6861)=-9.16, p<.001)$, and gender $(\beta=-$ $5.17, t(6861)=-8.02, p<.001)$ predicted number of items added, while device operating system $\operatorname{did} \operatorname{not}(\beta=.76, t(6861)=.97, p=.33)$. Looking at net sales, the overall model $(F(4,6861)=5.85$, $p<.001)$ was significant such that vibration condition $(\beta=3.26, t(6861)=2.00, p<.05)$ significantly predicted sales, while age was no longer significant $(\beta=.04, t(6861)=.59, p=.56)$, and gender was only marginally significant $(\beta=-3.16, t(6861)=-1.82, p=.07)$. In this model, device OS was a significant predictor $(\beta=8.15, t(6861)=2.10, p<.001)$, such that Android customers made orders with higher net sales. 


\section{Discussion}

The findings of this large-scale field experiment offer several key insights. First, this study highlights the managerial relevance of the current findings, providing initial evidence for how and why firms such as Amazon may have begun implementing mobile vibrations in ecommerce contexts. Second, the results corroborate our previous empirical study (Study 3a), demonstrating that mobile vibrations can influence shopping basket item adds in complex online mobile shopping environments with real economic consequences. Finally, we found that vibration still significantly affects the number of items purchased and sales even after accounting for differences in age, gender, and device operating system. As previously noted, we generally observed smaller effect size due to the uncontrollable nature of the field study. However, despite the smaller effect sizes indicating a more subtle but systematic effect on behavior, we highlight that this was a real-world field study, leading to small but systematic effects with real economic implications.

\section{STUDY 4}

We previously demonstrated (Study 1 and 3a) that prior exposure to positively valenced mobile events is a key moderator of the effect of mobile vibration on reward response and related consumer outcomes. Study 4 was designed to provide causal evidence for our proposed conditioning theory that vibrations are parsed as rewards due to their association with positively valenced mobile events. We test this theory by manipulating the valence of the mobile event associated with a neutral vibration then examining the process by which this differential conditioning affects the same consumer outcomes (item adds and sales), taking into account induced affect and the moderating role of impulsivity. 
Design and Procedure

We recruited 230 participants from an online panel for a between-subjects study. Eight participants failed the attention check, and a further three were removed for completing the study too quickly (more than three standard deviations below the mean duration completion time of 22.42 minutes $)$, leaving $219\left(M_{\mathrm{Age}}=36.86,47 \%\right.$ male $)$ as the final sample. After a hardware precheck and device handling instructions, as previously described, participants were randomly assigned to complete one of two versions of a Multi-arm Bandit (MAB) conditioning task.

Multi-arm bandit (MAB) conditioning task. The MAB task is a classic reinforcement learning problem, drawn from an imagined scenario of a row of slot machines (each one being a "one-armed bandit") from which a gambler must choose (Weber 1992). We created our two condition, web browser-based MABs by adapting an existing paradigm (Stojic et al. 2018) to a mobile touchscreen environment. Specifically, we presented participants in both conditions with four visually identical slot machines, each labeled with a randomly assigned letter. We informed participants that the machines had different probabilities of winning and that their goal in the game was to win as many points as possible, across several rounds. After each round, the machines and labels were shuffled so that participants had to relearn which machine was best in each round.

Participants were randomly assigned to either a positive valence condition, designed to condition participants to associate a neutral vibration (described in next paragraph) with a reward (i.e., positive valence), or to a negative valence condition, designed to condition participants to associate the same vibration with a loss (i.e., negative valence). In the positive valence condition, participants received a vibration each time they won, and no vibration when they lost. In the 
negative valence condition the opposite was true: participants received a vibration each time that they lost, and no vibration when they won. The task parameters for each condition, as well as illustrations of exemplar trials, are provided in Web Appendix A2.4.

In an attempt to mitigate the effects of prior exposure (Brown and Jenkins 1968; Pavlov 1932) to mobile vibration, we used a $100 \mathrm{~ms}$ vibration as our unconditioned stimulus because our previous studies demonstrated that $100 \mathrm{~ms}$ is both relatively neutral $(4.00=$ neutral $)$ in terms of reward $\left(M_{100 \mathrm{msReward}}=4.10\right)$ and not very familiar $\left(M_{100 \mathrm{msFamiliarity}}=4.14\right)$. In order to promote task engagement, we also made the MAB tasks incentive compatible by offering bonuses to the highest performing participants (detail in Web Appendix A2.4). Directly after the MAB task, participants completed a MAB Reward Response Questionnaire, which included items measuring MAB task reward response, adapted from the composite reward response measure previously described (e.g., "The slot machine task was rewarding”; see Web Appendix A1.8).

Online grocery shopping task. The Study 4 online grocery shopping task was the same as that used in Study 3a, except that adding an item to the shopping cart produced a $100 \mathrm{~ms}$ vibration in both conditions. After the shopping task, participants completed a 20 -item version of the Positive and Negative Affect Schedule (PANAS) oriented to evaluate the grocery shopping task (e.g., "During the grocery shopping simulation, to what extent did you feel [excited]?", $\alpha=.88$, adapted from Watson, Clark, and Tellegen 1988; see Web Appendix A1.10). We anticipated that participants conditioned to associate a $100 \mathrm{~ms}$ vibration with a reward would have a subsequently stronger reward response to that vibration. This rewarding response should then translate into greater positive affect in the shopping task, which in turn should lead to more item adds, relative to participants conditioned to associate the same vibration with a loss. 
Post-task measurements. We included a battery of self-report measures from previous studies. Specifically, participants completed the: Mobile Vibration Questionnaire, Mobile Usage Questionnaire, Barratt's Impulsiveness Scale, and a demographic questionnaire (all identical as previously described).

Results

Providing initial evidence of valence conditioning efficacy, we found that participants in the positive valence condition had a stronger reward response to the MAB task than did participants in the negative valence condition $\left(M_{\text {PosVibrationMAB-Reward }}=5.50\right.$ vs. $M_{\text {NegVibrationMAB- }}$ Reward $=2.37 ; F(1,217)=231.60, p<.001)$. Next, looking at whether the MAB vibration condition influenced subsequent response to the neutral $100 \mathrm{~ms}$ vibration, we find a main effect of MAB vibration condition on vibration reward response, such that participants in the positive valence condition had a marginally stronger reward response compared to participants in the negative valence condition $\left(M_{\text {PosValencel } 100 \mathrm{~m} s R e w a r d}=3.21\right.$ vs. $M_{\text {NegValencel } 100 \mathrm{msReward}}=2.76 ; F(1,217)=2.94$, $p=.09$ ). As noted, both previous exposure to mobile vibrations and the general notification valence influence mobile vibration response (Study 1, Study 2 and Study 3a) and could therefore dampen the effect of our brief conditioning task. After controlling for prior exposure and general notification valence, the effect of MAB condition on vibration reward response increased in magnitude $(F(1,215)=3.65, p=.05)$. Critically, the effect of the mobile vibration conditioning was also borne out in the subsequent shopping task: consumers in the positive valence condition added significantly more items to their shopping cart compared to participants in the negative valence condition $\left(M_{\text {PosValenceItemCount }}=30.26\right.$ vs. $M_{\text {NegValenceltemCount }}=26.28 ; F(1,213)=4.87$, $\left.p<.05, \eta^{2}=.02\right)$. Participants in the positive valence condition also had significantly higher 
basket totals $\left(M_{\text {PosValenceBasketTotal }}=\$ 158.13\right.$ vs. $M_{\text {NegValenceBasketTotal }}=\$ 126.37 ; F(1,213)=9.62$, $\left.p<.01, \eta^{2}=.04\right)$.

Vibration conditioning process. Next, we estimated a mediation model to examine the process by which the vibration conditioning task affected reward response and subsequently item count, taking into account the previously observed moderating role of impulsivity. Specifically, we estimated a serial moderated mediation model with 5,000 bootstrapped samples (Model 91; Hayes 2017) using MAB task reward response as the first mediator, shopping task positive affect as the second mediator, and impulsivity as a moderator between these mediators. Mirroring the main effect findings, the mediation model indicates that positive valence vibration reward conditioning was associated with a significantly stronger MAB reward response $\left(\beta_{\text {Condition }}=2.85\right.$, $t=15.31, p<.001)$. This stronger reward response led to a significant increase in positive affect in the shopping task $\left(\beta_{\text {MABReward }}=5.32, t=1.71, p<.001\right)$. Impulsivity moderated the effect of $\mathrm{MAB}$ reward response on shopping task positive affect $\left(\beta_{\mathrm{MABReward}} \mathrm{x}\right.$ Impulsivity $=-.17, t=-2.04$, $p<.05)$. Finally, this increased positive affect while shopping was associated with greater item count $\left(\beta_{\text {ShoppingPosAffect }}=1.17, t=3.07, p<.01\right)$. This model produced an overall significant moderated sequential mediation index ( $\beta_{\text {Indirect }}=-.15,95 \%$ CI:[- $\left.0.35,-.02\right]$ ). A process model of the same structure also produced a significant moderated mediation index when using basket total as the outcome variable ( $\beta_{\text {Indirect }}=-.58,95 \%$ CI: $\left.[-1.39,-.04]\right)$. 
Figure 7. Process model of how vibration conditioning influences item count (Study 4)

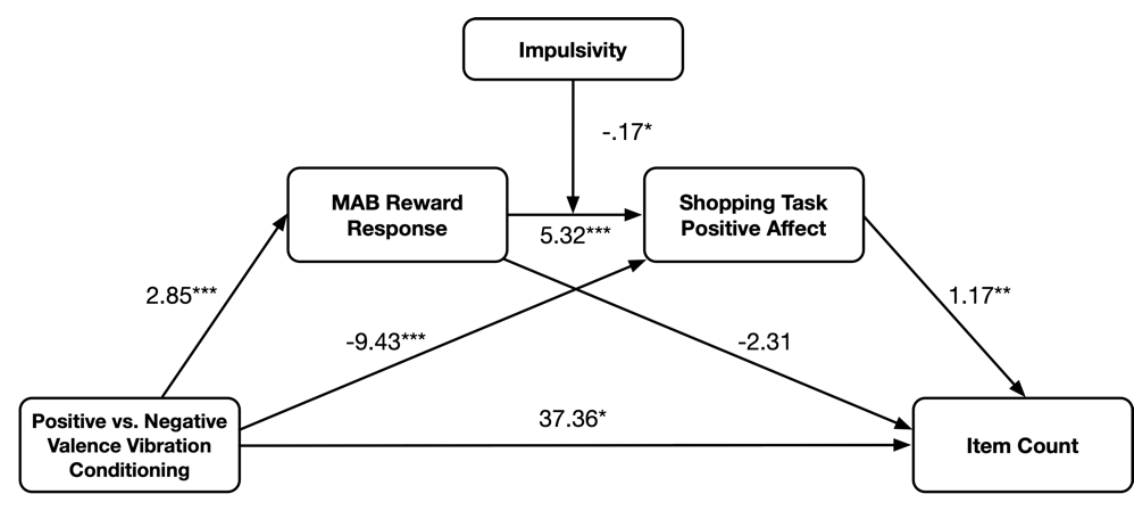

Notes: $\mathrm{MAB}=$ Multi-Armed Bandit; ${ }^{* * *}=p<.001,{ }^{* *}=p<.01,{ }^{*}=p<.05$.

Discussion

In Study 4 we designed a task to approximate the stages of a classical conditioning process (Figure 1), simulating the repeated pairing of neutral mobile vibration with a positively valenced mobile event. Specifically, we conditioned participants to associate a neutral mobile vibration with either a positively valenced reward or a negatively valenced loss using modified versions of a classic MAB reward learning task. We observed that participants who were conditioned to associate the vibration with a positively valenced event exhibited a heightened reward response, which boosted positive affect during shopping and ultimately lead to more purchased items and higher basket totals. These findings provide causal evidence supporting the hypothesis that consumers find mobile vibrations rewarding due to prior classical conditioning. We also provide further evidence on the moderating role of impulsivity, showing that greater impulsivity leads to a stronger reward response to mobile vibration. In a follow-up experiment (fully reported in Web Appendix C2), we directly manipulated impulsivity between-subjects, 
demonstrating that higher (vs. lower) induced impulsivity amplifies reward response to mobile vibrations, and subsequently boosts of the number of items added during a shopping task.

\section{GENERAL DISCUSSION}

Across five studies, we showed that pairing a rewarding mobile vibration (400ms; Study 1) with a mobile behavior increases the frequency of that behavior-from adding abstract items to a box (Study 2) to purchasing decisions in both laboratory-controlled (Study 3a) and realworld e-commerce contexts (Study 3b). We further showed that not all vibrations are created equal: the influence of mobile vibration is dependent on it evoking a reward response, which is a function of the valence of an associated mobile event (Study 4). We also demonstrated that mobile vibration has a heterogenous effect, influencing more strongly impulsive consumers and those who are generally more receptive to their mobile notifications. Despite these cohort differences, we found vibration durations of intermediate length $(400 \mathrm{~ms})$ consistently evoked the strongest reward response and accordingly most strongly influenced behavior.

Theoretical implications. The findings of this research make four primary contributions. First, the current studies respond to recent calls that consumer research has not kept pace with the rapidly expanding range of device types and interaction modalities (Stephen 2016; Yadav and Pavlou 2014). The current work provides a rigorous assessment of how mobile vibrations systematically influence reward response and alter consumer decision-making in online shopping contexts. While most previous studies of vibration have focused on ergonomics, information systems, and communication messages (Cole et al. 2015; Hadi and Valenzuela 2019; Lee and Lim 2014; Saket et al. 2013), we show how specific mobile vibrations can influence consumer 
decision-making, from adding more items to a box in abstract behavioral tasks, to adding more items to a real online shopping basket.

Second, the current work contributes to the reward literature, expanding what we know about how humans learn and process rewards through mobile interfaces. We know that people parse certain abstract stimuli such as paper money and power as rewards (Sescousse et al. 2013), and that touch serves as reward cue in infants (Blass, Ganchrow, and Steiner 1984), but the current study represents the first evidence that consumers interpret mobile vibration as a subtle secondary reward that can systematically influence consumer decision-making. We examine the genesis and process of this response, formally testing the theory that mobile vibrations can evoke a reward response due to classical conditioning (Study 4). Consistent with our theorizing, we demonstrate that conditioning consumers to associate a previously neutral vibration with a positively valenced mobile event leads consumers to exhibit a reward response to that vibration, which can then magnify mobile consumer actions including shopping cart adds. We provide evidence underscoring the importance of this vibration-linked notification valence using both moderation (Study 1 and 2) and causal mediation paradigms (Study 4). As a final examination of the importance of vibration exposure, we pooled our data across studies and confirmed that participants who experience very few daily vibrations do not even find intermediate vibrations rewarding, while those experiencing many vibrations found the same vibrations more rewarding (see Web Appendix D1.3 Figure 2). This provides further support for our conditioning theory, showing that in order for mobile vibrations to evoke a reward response, a certain amount of exposure is required.

Third, our findings extend work on the "psychology of touch" in marketing and consumer research. The majority of prior work on the "mobile consumer" has related to phenomena 
stemming from the differing touch modalities of mobile interfaces (Brasel and Gips 2015; Melumad, Inman, and Pham 2019; Shen et al. 2016; Stephen 2016). In contrast, the current research highlights the influence of haptic feedback in shaping consumer decision-making, demonstrating that even when touch modality is kept constant, mobile vibrations lead consumers to prefer mobile actions that are paired with a rewarding vibration. Our findings also supplements research on interpersonal touch demonstrating that different types and lengths of interpersonal touch result in differential subjective response (Hertenstein et al. 2009). Our studies extend this research into the vibration domain by demonstrating a number of novel functional relationships between mobile vibration duration and subjective response (such as reward response, which had an inverted-u shape). Finally, while preliminary evidence suggested that mobile vibrations could be positively valenced (Yoo et al. 2015), the current research integrates these previously disconnected streams of research by showing that specific mobile vibrations are construed as rewards that influence consumer behavior.

Fourth, our findings underscore the importance of trait-level differences in impulsivity and notification valence when studying consumer decision-making on mobile devices. We provide evidence across our studies that more impulsive consumers are more prone to the influence of mobile vibrational stimuli. Our studies also replicate and extend recent correlational findings evincing positive relationships between impulsivity, mobile usage, and reward responsiveness (Wilmer et al. 2019; Wilmer and Chein 2016a) by providing causal evidence that inducing higher impulsivity magnifies vibration reward response and subsequently induces higher spending (Study 4 Follow-up; Web Appendix C1). This finding suggests that a positive feedback loop may underlie mobile technology engagement: initial trait levels of impulsivity lead to higher engagement with mobiles and reward-seeking behavior that is reinforced by 
mobile use, which leads to further impulsivity induction. In addition to impulsivity, we also found that consumers with more positively valenced mobile notifications responded more strongly to the influence of mobile vibration (Study 1 and 3a). Given this effect was stronger in younger consumers (Web Appendix D1.2. Table 1), our findings have particular gravity given that this younger demographic constitutes a behaviorally distinct and rapidly growing group of ecommerce consumers (Moore 2012; SivaKumar and Gunasekaran 2017; Wood 2013).

Managerial implications. The current findings have immediate implications for managers. We found that mobile vibrations can be used to boost specific mobile actions. In these studies, we used vibrations to promote generic mobile clicking behavior (Study 2), as well as to increase number of items purchased in both laboratory-controlled (Study 3a) and real-world consumptive e-commerce settings (Study 3b). These findings are expected to further increase in importance given that mobile spending is growing over three times faster than desktop spending (Comscore 2020a), particularly for online grocery shopping which has outstripped apparel as the number one source of digital spending (Comscore 2020b). Given our finding that adding a mobile vibration to shopping cart adds boosted item adds by between $3 \%$ to $18 \%$, our results indicate that mobile vibration feedback offers a straightforward way to increase sales in online mobile shopping contexts. Our results also suggest that such vibrational stimuli could be used to boost other behaviors - theoretically any behavior that can be paired with a mobile vibration might be bolstered. Such vibrational feedback could easily be implemented in both mobile applications and mobile websites.

Conclusion. In sum, the current research demonstrates that haptic feedback can robustly shape consumer preference and choice. We show that pairing an intermediate length vibration with a specific behavior, such as adding items to a shopping cart, boosts the frequency of that 
behavior. We provide evidence on the underlying psychological mechanism consistent with a classic conditioning process that renders vibrations rewarding due to their learned association with positively valenced mobile notifications. The findings of this research contribute to foundational work examining how haptic human-machine interactions affect the modern, technology-augmented consumer, the effective design of haptic technologies in marketing, and how subtle vibrations on mobile devices are systematically altering the way we search, shop, and express our preferences. 


\section{References}

van den Akker, Karolien, Anita Jansen, Florentine Frentz, and Remco C Havermans (2013), “Impulsivity Makes More Susceptible to Overeating after Contextual Appetitive Conditioning," Appetite, 70, 73-80.

Beatty, Sharon E and M Elizabeth Ferrell (1998), "Impulse Buying: Modeling Its Precursors," Journal of retailing, 74(2), 169-91.

Van den Berg, Ivo, Ingmar H A Franken, and Peter Muris (2010), “A New Scale for Measuring Reward Responsiveness," Frontiers in psychology, 1, 239.

Berridge, Kent C (2000), "Reward Learning: Reinforcement, Incentives, and Expectations," in Psychology of Learning and Motivation, Elsevier, 223-78.

Blass, Elliott M, Judith R Ganchrow, and Jacob E Steiner (1984), “Classical Conditioning in Newborn Humans 2-48 Hours of Age," Infant behavior and development, 7(2), 223-35.

Bovenzi, Massimo and Antonella Zadini (1992), "Self-Reported Low Back Symptoms in Urban Bus Drivers Exposed to Whole-Body Vibration.," Spine, 17(9), 1048-59.

Brasel, S Adam and James Gips (2015), “Interface Psychology: Touchscreens Change Attribute Importance, Decision Criteria, and Behavior in Online Choice," Cyberpsychology, Behavior, and Social Networking, 18(9), 534-38.

- (2014), “Tablets, Touchscreens, and Touchpads: How Varying Touch Interfaces Trigger Psychological Ownership and Endowment," Journal of Consumer Psychology, 24(2), 22633.

Brown, Lorna M and Topi Kaaresoja (2006), “Feel Who’s Talking: Using Tactons for Mobile Phone Alerts," in CHI'06 Extended Abstracts on Human Factors in Computing Systems, 
ACM, 604-9.

Brown, Paul L and Herbert M Jenkins (1968), “AUTO-SHAPING OF THE PIGEON'S KEYPECK 1," Journal of the experimental analysis of behavior, 11(1), 1-8.

Brown, Sarah M, Stephen B Manuck, Janine D Flory, and Ahmad R Hariri (2006), "Neural Basis of Individual Differences in Impulsivity: Contributions of Corticolimbic Circuits for Behavioral Arousal and Control.,"Emotion, 6(2), 239.

Carver, Charles S. and Teri L. White (1994), "Behavioral Inhibition, Behavioral Activation, and Affective Responses to Impending Reward and Punishment: The BIS/BAS Scales," Journal of Personality and Social Psychology, 67(2), 319-33, http://doi.apa.org/getdoi.cfm?doi=10.1037/0022-3514.67.2.319.

Cheng, Yin-Hui, Shih-Chieh Chuang, Sui-Min Wang, and Shan-yun Kuo (2013), "The Effect of Companion's Gender on Impulsive Purchasing: The Moderating Factor of Cohesiveness and Susceptibility to Interpersonal Influence," Journal of Applied Social Psychology, 43(1), $227-36$.

Cole, Alan G, Siddique A Mohammed, and Dhandapani Shanmugam (2015), "Providing a Sense of Touch in a Mobile Device Using Vibration."

Comscore (2020a), “Comscore's 2020 State of Retail,” https://www.comscore.com/Insights/Events-and-Webinars/Webinar/2020/State-of-Retail. (2020b), “Grocery Is Top Mobile Spending Category for First Time," https://www.comscore.com/Insights/Blog/Grocery-is-Top-Mobile-Spending-Category-forFirst-Time.

Constine, Josh (2014), “Pavlok’s Wristband That Electroshocks You For Facebooking Or 
Skipping Workouts Now On Indiegogo,” TechCrunch, https://techcrunch.com/2014/09/30/pavlok/.

Crusco, April H and Christopher G Wetzel (1984), "The Midas Touch: The Effects of Interpersonal Touch on Restaurant Tipping," Personality and Social Psychology Bulletin, 10(4), 512-17.

Davenport, Kate, James E Houston, and Mark D Griffiths (2012), “Excessive Eating and Compulsive Buying Behaviours in Women: An Empirical Pilot Study Examining Reward Sensitivity, Anxiety, Impulsivity, Self-Esteem and Social Desirability," International Journal of Mental Health and Addiction, 10(4), 474-89.

Drouin, Michelle, Daren H Kaiser, and Daniel A Miller (2012), “Phantom Vibrations among Undergraduates: Prevalence and Associated Psychological Characteristics," Computers in Human Behavior, 28(4), 1490-96.

Elhai, Jon D, Jason C Levine, Robert D Dvorak, and Brian J Hall (2016), "Fear of Missing out, Need for Touch, Anxiety and Depression Are Related to Problematic Smartphone Use," Computers in Human Behavior, 63, 509-16.

Gastwirth, Joseph L, Yulia R Gel, and Weiwen Miao (2009), “The Impact of Levene's Test of Equality of Variances on Statistical Theory and Practice," Statistical Science, 24(3), 34360.

Gelman, Andrew and Jennifer Hill (2007), "Data Analysis Using Regression and Hierarchical/Multilevel Models," New York, NY: Cambridge.

Gescheider, George A, Marian E Berryhill, Ronald T Verrillo, and Stanley J Bolanowski (1999), "Vibrotactile Temporal Summation: Probability Summation or Neural Integration?," 
Somatosensory \& motor research, 16(3), 229-42.

Gormezano, Isidore and J W Moore (1966), “Classical Conditioning,” Experimental methods and instrumentation in psychology, 1, 385-420.

Green, Barry G (1976), “Vibrotactile Temporal Summation: Effect of Frequency.," Sensory Processes.

Gupta, Sunita (1990), "Impulsivity/Sociability and Reinforcement in Verbal Operant Conditioning: A Replication," Personality and Individual Differences, 11(6), 585-89.

Hadar, A., D. Eliraz, A. Lazarovits, U. Alyagon, and A. Zangen (2015), “Using Longitudinal Exposure to Causally Link Smartphone Usage to Changes in Behavior, Cognition and Right Prefrontal Neural Activity," Brain Stimulation, 8(2), 318, http://linkinghub.elsevier.com/retrieve/pii/S1935861X15004568.

Hadi, Rhonda and Ana Valenzuela (2019), “Good Vibrations: Consumer Responses to Technology-Mediated Haptic Feedback," Journal of Consumer Research.

Hampton, William H., Kylie H. Alm, Vinod Venkatraman, Tehila Nugiel, and Ingrid R. Olson (2017), “Dissociable Frontostriatal White Matter Connectivity Underlies Reward and Motor Impulsivity,” NeuroImage, 150(July 2016), 336-43, http://dx.doi.org/10.1016/j.neuroimage.2017.02.021.

Hayes, Andrew F (2017), Introduction to Mediation, Moderation, and Conditional Process Analysis: A Regression-Based Approach, Guilford publications.

Hertenstein, Matthew J, Rachel Holmes, Margaret McCullough, and Dacher Keltner (2009), “The Communication of Emotion via Touch.," Emotion, 9(4), 566.

Huber, Peter J (1973), “Robust Regression: Asymptotics, Conjectures and Monte Carlo," The 
Annals of Statistics, 1(5), 799-821.

Kaaresoja, Topi and Jukka Linjama (2005), "Perception of Short Tactile Pulses Generated by a Vibration Motor in a Mobile Phone," in First Joint Eurohaptics Conference and Symposium on Haptic Interfaces for Virtual Environment and Teleoperator Systems. World Haptics Conference, IEEE, 471-72.

Kelley, Ann E (2004), "Ventral Striatal Control of Appetitive Motivation: Role in Ingestive Behavior and Reward-Related Learning," Neuroscience \& biobehavioral reviews, 27(8), $765-76$.

Koskinen, Emilia, Topi Kaaresoja, and Pauli Laitinen (2008), "Feel-Good Touch: Finding the Most Pleasant Tactile Feedback for a Mobile Touch Screen Button," in Proceedings of the 10th International Conference on Multimodal Interfaces, ACM, 297-304.

Krishna, Aradhna and Maureen Morrin (2007), "Does Touch Affect Taste? The Perceptual Transfer of Product Container Haptic Cues," Journal of Consumer Research, 34(6), 80718.

Krishna, Aradhna and Norbert Schwarz (2014), "Sensory Marketing, Embodiment, and Grounded Cognition: A Review and Introduction,” Journal of consumer psychology, 24(2), $159-68$.

Lee, Heow Pueh and Siak Piang Lim (2014), “Comparative Studies of Perceived Vibration Strength for Commercial Mobile Phones," Applied ergonomics, 45(3), 807-10.

Mehrotra, Abhinav, Mirco Musolesi, Robert Hendley, and Veljko Pejovic (2015), “Designing Content-Driven Intelligent Notification Mechanisms for Mobile Applications," in Proceedings of the 2015 ACM International Joint Conference on Pervasive and Ubiquitous 
Computing, ACM, 813-24.

Melumad, Shiri, J. Jeffrey Inman, and Michel Tuan Pham (2019), "Selectively Emotional: How Smartphone Use Changes User-Generated Content," Journal of Marketing Research.

Melumad, Shiri and Michel Tuan Pham (2020), "The Smartphone as a Pacifying Technology," Journal of Consumer Research.

Moore, Marguerite (2012), “Interactive Media Usage among Millennial Consumers,” Journal of Consumer Marketing, 29(6), 436-44.

Olson, Michael A and Russell H Fazio (2001), "Implicit Attitude Formation through Classical Conditioning," Psychological Science, 12(5), 413-17.

Park, C Whan and V Parker Lessig (1981), "Familiarity and Its Impact on Consumer Decision Biases and Heuristics," Journal of consumer research, 8(2), 223-30.

Patton, Jim H., Matthew S. Stanford, and Ernest S. Barratt (1995), "Factor Structure of the Barratt Impulsiveness Scale,” Journal of Clinical Psychology, 51(6), 768-74.

Pavlov, Ivan Petrovich (1932), "The Reply of a Physiologist to Psychologists."

Peck, Joann and Terry L. Childers (2003), "Individual Differences in Haptic Information Processing: The ‘Need for Touch' Scale,” Journal of Consumer Research, 30(3), 430-42.

Peck, Joann and Terry L Childers (2003), "To Have and to Hold: The Influence of Haptic Information on Product Judgments," Journal of Marketing, 67(2), 35-48.

Peck, Joann and Suzanne B Shu (2009), "The Effect of Mere Touch on Perceived Ownership," Journal of consumer Research, 36(3), 434-47.

Peck, Joann and Jennifer Wiggins (2006), “It Just Feels Good: Customers’ Affective Response to 
Touch and Its Influence on Persuasion," Journal of Marketing, 70(4), 56-69.

Peppet, Scott R (2014), “Regulating the Internet of Things: First Steps toward Managing Discrimination, Privacy, Security and Consent," Tex. L. Rev., 93, 85.

Puri, Radhika (1996), “Measuring and Modifying Consumer Impulsiveness: A Cost-Benefit Accessibility Framework.," Journal of consumer Psychology.

Rosenberger, Robert (2015), “An Experiential Account of Phantom Vibration Syndrome,” Computers in Human Behavior, 52, 124-31.

Sahami, Alireza, Paul Holleis, Albrecht Schmidt, and Jonna Häkkilä (2008), "Rich Tactile Output on Mobile Devices," in European Conference on Ambient Intelligence, Springer, $210-21$.

Saket, Bahador, Chrisnawan Prasojo, Yongfeng Huang, and Shengdong Zhao (2013), "Designing an Effective Vibration-Based Notification Interface for Mobile Phones," in Proceedings of the 2013 Conference on Computer Supported Cooperative Work, ACM, $149-1504$.

Schag, Kathrin, Martin Teufel, Florian Junne, Hubert Preissl, Martin Hautzinger, Stephan Zipfel, and Katrin Elisabeth Giel (2013), "Impulsivity in Binge Eating Disorder: Food Cues Elicit Increased Reward Responses and Disinhibition,” PloS one, 8(10), e76542.

Seifi, Hasti and Karon E Maclean (2013), “A First Look at Individuals’ Affective Ratings of Vibrations," in 2013 World Haptics Conference (WHC), IEEE, 605-10.

Sescousse, Guillaume, Xavier Caldú, Bàrbara Segura, and Jean-Claude Dreher (2013), "Processing of Primary and Secondary Rewards: A Quantitative Meta-Analysis and Review of Human Functional Neuroimaging Studies," Neuroscience \& Biobehavioral Reviews, 
37(4), 681-96.

Shankar, Venkatesh, Alladi Venkatesh, Charles Hofacker, and Prasad Naik (2010), "Mobile Marketing in the Retailing Environment: Current Insights and Future Research Avenues,” Journal of interactive marketing, 24(2), 111-20.

Shen, Hao, Meng Zhang, and Aradhna Krishna (2016), "Computer Interfaces and the 'DirectTouch' Effect: Can IPads Increase the Choice of Hedonic Food?," Journal of Marketing Research, In-Press.

Sherman, Lauren E., Ashley A. Payton, Leanna M. Hernandez, Patricia M. Greenfield, and Mirella Dapretto (2016), “The Power of the Like in Adolescence: Effects of Peer Influence on Neural and Behavioral Responses to Social Media," Psychological Science, 27(7), 102735, http://pss.sagepub.com/lookup/doi/10.1177/0956797616645673.

Shu, Suzanne B. and Joann Peck (2011), "Psychological Ownership and Affective Reaction: Emotional Attachment Process Variables and the Endowment Effect," Journal of Consumer Psychology, 21, 439-52.

Silver, Lauren and Stefan Cornibert (2019), Smartphone Ownership Is Growing Rapidly Around the World, but Not Always Equally, Pew Research Center.

Simon, Joe J, Stephan Walther, Christian J Fiebach, Hans-Christoph Friederich, Christoph Stippich, Matthias Weisbrod, and Stefan Kaiser (2010), "Neural Reward Processing Is Modulated by Approach-and Avoidance-Related Personality Traits," Neuroimage, 49(2), $1868-74$.

SivaKumar, ArunKumar and Abirami Gunasekaran (2017), "An Empirical Study on the Factors Affecting Online Shopping Behavior of Millennial Consumers," Journal of Internet 
Commerce, 16(3), 219-30.

Steinberg, Laurence, Dustin Albert, Elizabeth Cauffman, Marie Banich, Sandra Graham, and Jennifer Woolard (2008), “Age Differences in Sensation Seeking and Impulsivity as Indexed by Behavior and Self-Report: Evidence for a Dual Systems Model,” Developmental Psychology, 44(6), 1764-78.

Stephen, Andrew T (2016), “The Role of Digital and Social Media Marketing in Consumer Behavior," Current Opinion in Psychology, 10, 17-21.

Stojic, Hrvoje, Eric Schulz, Pantelis P Analytis, and Maarten Speekenbrink (2018), “It’s New, but Is It Good? How Generalization and Uncertainty Guide the Exploration of Novel Options."

Thissen, David, Lynne Steinberg, and Daniel Kuang (2002), "Quick and Easy Implementation of the Benjamini-Hochberg Procedure for Controlling the False Positive Rate in Multiple Comparisons," Journal of educational and behavioral statistics, 27(1), 77-83.

Vanhemert, Kyle (2015), “Apple's Haptic Tech Is a Glimpse at the UI of the Future,” Wired.

Verrillo, R T (1965), “Temporal Summation in Vibrotactile Sensitivity," The Journal of the Acoustical Society of America, 37(5), 843-46.

Watson, David, Lee Anna Clark, and Auke Tellegen (1988), "Development and Validation of Brief Measures of Positive and Negative Affect: The PANAS Scales.," Journal of personality and social psychology, 54(6), 1063.

Weber, Richard (1992), "On the Gittins Index for Multiarmed Bandits," The Annals of Applied Probability, 2(4), 1024-33.

Wilmer, Henry H. and Jason M. Chein (2016a), “Mobile Technology Habits: Patterns of 
Association among Device Usage, Intertemporal Preference, Impulse Control, and Reward Sensitivity," Psychonomic Bulletin \& Review, 1-8.

(2016b), "Mobile Technology Habits:Â Patterns of Association among Device Usage, Intertemporal Preference, Impulse Control, and Reward Sensitivity," Psychonomic Bulletin and Review, 23(5), 1607-14.

Wilmer, Henry H., William H. Hampton, Thomas M. Olino, Ingrid R. Olson, and Jason M. Chein (2019), "Wired to Be Connected? Links between Mobile Technology Engagement, Intertemporal Preference and Frontostriatal White Matter Connectivity," Social Cognitive and Affective Neuroscience, 14(4), 367-79.

Wolfe, Jeremy M, Keith R Kluender, Dennis M Levi, Linda M Bartoshuk, Rachel S Herz, Roberta L Klatzky, Susan J Lederman, and D M Merfeld (2006), Sensation \& Perception, Sinauer Sunderland, MA.

Wood, Stacy (2013), “Generation Z as Consumers: Trends and Innovation,” Institute for Emerging Issues: NC State University, 1-3.

Yadav, Manjit S and Paul A Pavlou (2014), "Marketing in Computer-Mediated Environments: Research Synthesis and New Directions," Journal of Marketing, 78(1), 20-40.

Yoo, Yongjae, Taekbeom Yoo, Jihyun Kong, and Seungmoon Choi (2015), “Emotional Responses of Tactile Icons: Effects of Amplitude, Frequency, Duration, and Envelope," in 2015 IEEE World Haptics Conference (WHC), IEEE, 235-40.

Zhou, Lianxi and Amy Wong (2004), "Consumer Impulse Buying and In-Store Stimuli in Chinese Supermarkets," Journal of International Consumer Marketing, 16(2), 37-53. 